\title{
Smooth Design of Broadband Beamformers Problem in Far Field
}

\author{
Mei Xiao ${ }^{2, \text { a) }}$, Dan $\mathrm{Li}^{2}$, b) , Zhiguo Feng ${ }^{1,2, c)}$ \\ ${ }^{1}$ Faculty of Mathematics and Computer Science, Guangdong Ocean University Zhanjiang, Guangdong 524088, \\ China. \\ ${ }^{2}$ College of Mathematics Science, Chongqing Normal University, Chongqing 401331, China. \\ a)775790466@qq.com, b) 253443281@qq.com, ${ }^{c)} 18281102 @ q q . c o m$
}

\begin{abstract}
The design of broadband beamformers can be formulated as a minimax problem such that the maximum error between the actual response function and a given desired response function in a specified domain is minimized. There is a transition region between the passband region and the stopband region, and the actual response oscillates in the transition region when the filter length increases. In this paper, we consider the smooth design of broadband beamformers such that there is no oscillation in the transition region, and the performance can also be remained.
\end{abstract}

Key words: Far field, Broadband beamformer, Transition region.

\section{INTRODUCTION}

Speech signal is one of the most common signals, and microphone is the device to receive and process the speech signal [1,2]. Then, microphone array model is widely used and has many practical applications such as hearing-aid, speech recognition, video conferencing, mobile phone etc. For microphone array, beamformer design is an important technique, which it can be applied to signal enhancement, blind signal separation [3] and acoustic localization. There are many optimization methods which can solve this problem such as linear semi-infinite programming, sequential quadratic programming [4], two-stage method [5], window function method [6]. However, the optimal solution always cause oscillation in transition region, where the actual response becomes very poor. For this, it is required to design a smooth response function in transition region, while the performance of the design can also be remained.

This paper is organized as follows. In Section 2, the optimal design of broadband beamformer in far field is introduced. In Section 3, a modified design of broadband beamformer in far field is formulated to have a smooth transition region. The smooth design is illustrated by an example in Section 4 with optimal design and modified design respectively.

\section{PROBLEM FORMULATION}

Suppose that there are $\mathrm{N}$ microphones, and for each microphone, there is a FIR filter behind. Then, the frequency response functions of FIR filters are

$$
\begin{gathered}
\boldsymbol{R}(f)=\left[R_{\mathrm{l}}(f), \cdots, R_{N}(f)\right]^{T}, \\
R_{i}(f)=\boldsymbol{w}_{i} g_{0}(f), \quad i=1, \cdots, N, \\
\boldsymbol{w}_{i}=\left[w_{i}(0), w_{i}(1), \cdots, w_{i}(L-1)\right]^{T},
\end{gathered}
$$


where $\mathrm{L}$ is filter length, and $g_{0}(f)$ is given by

$$
g_{0}(f)=\left[1, e^{-j 2 \pi f / f_{s}}, \cdots, e^{-j 2 \pi f(L-1) / f_{s}}\right],
$$

where $f_{s}$ is the sampling rate. The transfer function of the microphone array is given by

$$
\boldsymbol{T}(f, \boldsymbol{\theta})=\left[T_{1}(f, \boldsymbol{\theta}), \cdots, T_{N}(f, \boldsymbol{\theta})\right]^{T},
$$

Where:

$$
T_{i}(f, \boldsymbol{\theta})=e^{-j 2 \pi f r_{i} \cos \theta / c}, \quad \boldsymbol{\theta} \in[0, \pi], \quad i=1, \cdots, N
$$

where $\theta \in[0, \pi], r_{i}$ is the spatial point of the $i$-th microphone, and $c$ is the sound's speed in air.

According to real applications, the actual response function $G(f, \theta)$ is required to fit to an ideal response function. In general, the ideal response function is given by:

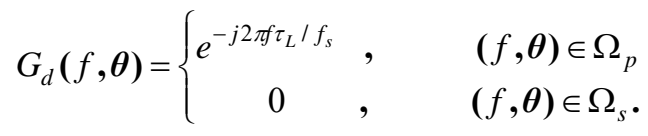

where $\Omega=\Omega_{p} \cup \Omega_{s}, \Omega_{p}$ is the passband region and $\Omega_{s}$ is the stopband region.

The cost function of broadband beamformer design is given by:

$$
\max _{(f, \theta) \in \Omega} \rho^{2}(f, \boldsymbol{\theta})\left|\boldsymbol{T}^{T}(f, \boldsymbol{\theta}) \boldsymbol{R}(f)-G_{d}(f, \boldsymbol{\theta})\right|,
$$

where $\rho(f, \boldsymbol{\theta})$ is a positive weighting function. Then, the beamformer design problem is to design $\boldsymbol{w}=\left[\boldsymbol{w}_{1}^{T}, \cdots, \boldsymbol{w}_{N}^{T}\right]$, such that the cost function above is minimized. This problem is equivalent to a semi-infinite programming problem as follows.

$$
\begin{array}{cc}
\min _{z, \boldsymbol{w}} & z \\
\text { s.t. } & H(\boldsymbol{w}, f, \boldsymbol{\theta}) \leq z, \quad \forall(f, \boldsymbol{\theta}) \in \Omega \\
& H(\boldsymbol{w}, f, \boldsymbol{\theta})=\rho^{2}(f, \boldsymbol{\theta})\left|T^{T}(f, \boldsymbol{\theta}) \boldsymbol{R}(f)-G_{d}(f, \boldsymbol{\theta})\right|^{2} .
\end{array}
$$

In general, only passband region and stopband region are cared in the cost function, that is, the performance of the design will not be affected in the transition region. Then, the actual response function may appear oscillation phenomenon in transition region.

In order to have a smooth actual response in the transition region, it requires a smooth ideal response function defined in the transition region. 


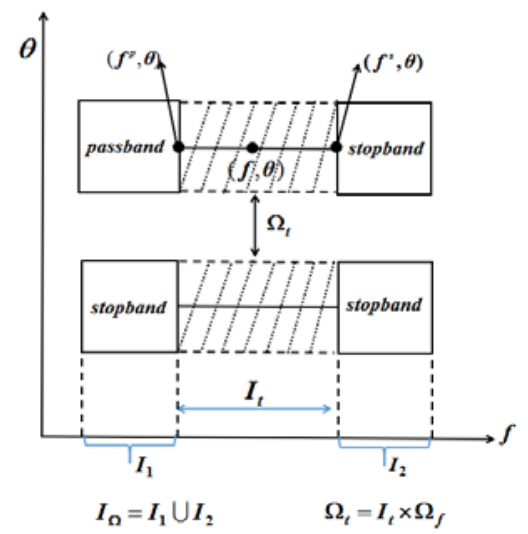

FIG. 1. The transition region

First, we decompose the frequency domain $\Omega$ as:

$$
\Omega=\bigcup_{f \in\left[0, f_{s} / 2\right]} \Omega_{f}
$$

where for each frequency $f, \Omega_{f}$ is the corresponding spatial domain of $f$ in $\Omega$. We denote the set of all frequencies $f$ satisfies $\Omega_{f} \neq \phi\left(\phi\right.$ denotes the empty set) in $\left[0, f_{s} / 2\right]$ by $I_{\Omega}$. The transition frequency is the frequency domain in $\left[0, f_{s} / 2\right]$ and not in $I_{\Omega}$, which can be denoted by $I_{t}$. Then, the transition region is denoted by $I_{t} \times \Omega_{f}$. Note that $I_{t}$ consists of several open intervals of endpoints belonging to $I_{\Omega}$. For a given $(f, \theta) \in I_{t} \times \Omega_{f}$, we can find two endpoints $\left(f^{p}, \boldsymbol{\theta}\right)$ and $\left(f^{s}, \boldsymbol{\theta}\right)$ (Fig.1). Then, we can define the value $G_{d}(f, \boldsymbol{\theta})$ by the interpolation of the function values in these two points.

Furthermore, the weighting function is to measure the important of the domain, therefore, we need to define the weight function in the transition region, and an appropriate weight function magnitude in transition region should be less than or equal to that in the specified region $\Omega$.

Then, the smooth beamformer design problem is formulated in to a modified design problem as:

$$
\begin{array}{ll}
\min _{z, \boldsymbol{w}} & z \\
\text { s.t. } & H(\boldsymbol{w}, f, \boldsymbol{\theta}) \leq z, \quad \forall(f, \boldsymbol{\theta}) \in \Omega \cup \Omega_{t} \\
& H(\boldsymbol{w}, f, \boldsymbol{\theta})=\rho^{2}(f, \boldsymbol{\theta})\left|T^{T}(f, \boldsymbol{\theta}) \boldsymbol{R}(f)-G_{d}(f, \boldsymbol{\theta})\right|^{2} .
\end{array}
$$

\section{NUMERICAL EXAMPLE}

To illustrate the problem, we have the following example, where

$$
\begin{gathered}
\Omega_{p}=\left\{(f, \boldsymbol{\theta}): f \in[0.3 \mathrm{kHz}, 2.0 \mathrm{kHz}], \boldsymbol{\theta} \in\left[80^{\circ}, 100^{\circ}\right]\right\}, \\
\Omega_{s}=\left\{(f, \boldsymbol{\theta}): f \in[0.3 \mathrm{kHz}, 2.0 \mathrm{kHz}] \bigcup[2.5 \mathrm{kHz}, 4.0 \mathrm{kHz}], \boldsymbol{\theta} \in\left[0^{\circ}, 40^{\circ}\right] \cup\left[120^{\circ}, 180^{\circ}\right]\right\} .
\end{gathered}
$$

The linear microphone array is placed parallel to the $\mathrm{x}$-axis, where there are 9 elements with space $0.05 \mathrm{~cm}$. The fifth microphone is in the center position, which is used as a reference point. The delay is $\tau_{L}=(L-1) / 2$. The other parameters are $c=340.9 \mathrm{~m} / \mathrm{s}, f_{\mathrm{s}}=8 \mathrm{kHz}$, and $\rho(f, \boldsymbol{\theta})=1, \boldsymbol{\theta} \in[0, \pi]$. 
After setting the filter length as 30, the optimal coefficients of the filters can be solved and the performance is obtained as $-14.7692 \mathrm{~dB}$. In order to show the shape of the actual response function, we plot the magnitude of the actual response function in Fig 2. It can be seen that the actual response function in transition region becomes bad.

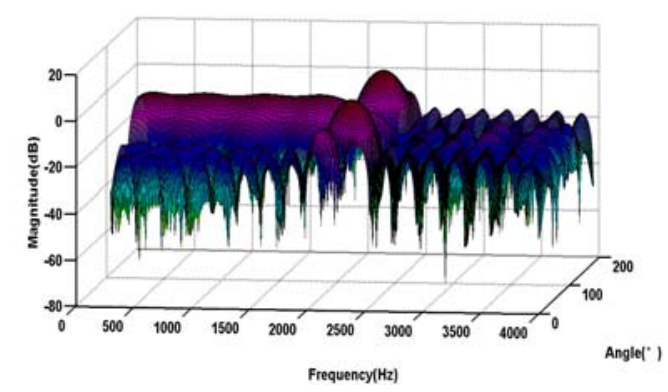

FIG. 2. Actual response function of optimal design

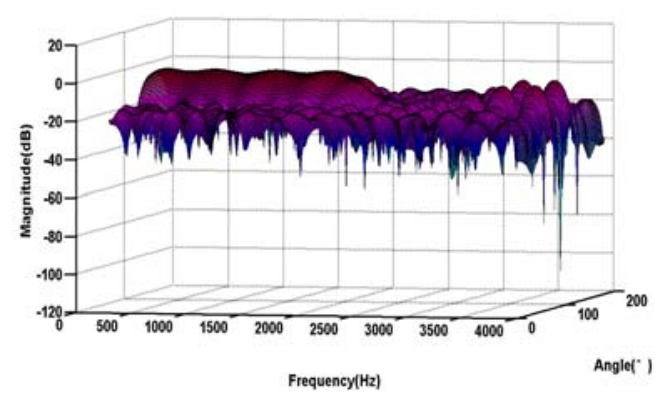

FIG.3.Actual response function of modified design

To improve the beamformer design, we modify the problem by defining the ideal response function in transition region as

$$
G_{d}(f, \boldsymbol{\theta})=\left\{\begin{array}{cl}
\frac{2.5 \mathrm{k}-f}{0.5 \mathrm{k}} e^{-j 2 \pi f \tau_{L} / \mathrm{f}_{\mathrm{s}}}, & (f, \boldsymbol{\theta}) \in \Omega_{f^{p}} \times I_{t}, \\
0 & ,(f, \boldsymbol{\theta}) \in \Omega_{f^{s}} \times I_{t},
\end{array}\right.
$$

Where

$$
\Omega_{f^{s}}=\left[0^{\circ}, 60^{\circ}\right] \cup\left[120^{\circ}, 180^{\circ}\right], \Omega_{f^{p}}=\left(80^{\circ}, 100^{\circ}\right), I_{t}=(2.0 \mathrm{kHz}, 2.5 \mathrm{kHz}) .
$$

Then, we set up the modified design problem and the optimal solution can be solved. The performance is obtained as $-13.4876 \mathrm{~dB}$, which is very close to that of optimal design. The magnitude of actual response function is depicted in Fig 3. It can be seen that the actual response becomes very smooth in the transition region.

Finally, we set the filter length from 5 to 30 and solve the optimal design problem and modified design problem, respectively. The performances are plotted in Fig 4. We can observe that the performance of the modified design is satisfied. 


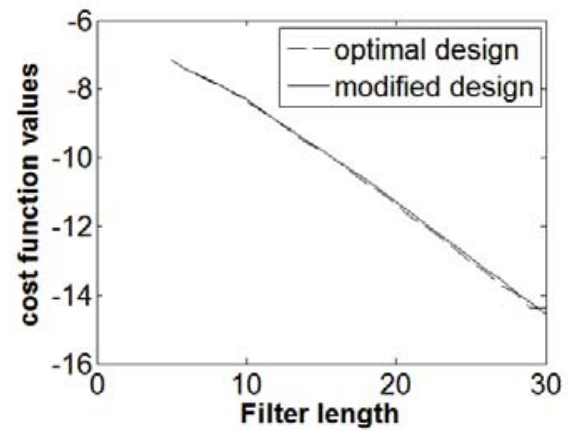

FIG. 4. Comparison of the cost function values with different filter lengths.

\section{SUMMARY}

In this paper, we consider the design of broadband beamformer in far field. By defining an adequate cost function in transition region and introducing the interpolation method, we formulate a modified design of broadband beamformer. We demonstrate by example that the improved smooth design can indeed suppress oscillation in the transition region, and the performance can be remained.

\section{ACKNOWLEDGEMENTS}

This paper is supported by the grant of National Natural Science Foundation of China (No. 61673078), the grant of Chongqing Science and Technology Commission (No. cstc2017jcyjAX0161), the grant of Chongqing Normal University (No. 17XLB010) and the graduate innovative research grant of Chongqing Normal University (No. YKC17016).

\section{REFERENCES}

1. R.C. Nongpiur. Design of minimax broadband beamformers that are robust to microphone gain, phase and position errors. IEEE Transactions on Audio Speech and Language Processing. Vol. 22(2014), p.1013-1022.

2. M. Brandstein, D.B. Ward. Microphone Arrays: Signal Processing Techniques and Applications. SpringerVerlag, Berlin, 2011.

3. J. Choi, C.C. Lim. A Cholesky factorization-based approach for blind FIR channel identification. IEEE Trans. Signal Processing. Vol. 56(2008), p.1730-1735.

4. S. Nordebo, I. Claesson, S. Nordholm. Weighted Chebyshev approximation for the design of broadband beamformers using quadratic programming. IEEE Signal Processing Letters. Vol. 1(1994), p. 103-105.

5. Z.G. Feng, K.F.C. Yiu, S. Nordholm. A two-stage method for the design of near-filed broadband beamformer. IEEE Trans. Signal Processing. Vol. 59(2011), p.3647-3656.

6. Z.G. Feng, K.F.C. Yiu. The design of multi-dimensional acoustic beamformers via window functions. Digital Signal Processing. Vol. 29(2014), p.107-116. 